effects on pericyte biology in vitro, yet the significance of this in vivo and in regard to diabetes remains unexplored.

Methods Using a mouse model with a pericyte specific knockout of the insulin receptor (PIR-/-), created by crossing PDGFR $\beta^{2}$-Cre mice with insulin receptor - floxed - mice, we investigated the role of pericyte insulin signalling in developmental retinal angiogenesis in vivo and on pericyte function in vitro.

Results At postnatal day 5, PIR-/- retinas show hyper-vascularized venous regions with an increase in venous vascular density $(57.6 \pm 1.0 \%$ compared to $52.1 \pm 0.6 \%$ in littermate controls, $\mathrm{p}=0.0003$ ) along with an increase in sprouting tip cell formation at the vascular front $(1.7 \pm 0.05$ versus 1.5 \pm 0.06 per $100 \mu \mathrm{m}$ in controls, $\mathrm{p}=0.006)$. Pericyte coverage at postnatal day 5 appears comparable in PIR-/- and control, but pericyte abundance is strikingly reduced by $20 \%$ in the mature adult retinal vasculature $(0.8 \pm 0.03$ and $1.0 \pm 0.05$ per $100 \mu \mathrm{m}$ in controls, $\mathrm{p}=0.002)$. However, the differential vascularity of neonatal retinas did not persist in adulthood $(18.1 \pm 0.7 \mathrm{~mm}$ versus $18.3 \pm 0.5 \mathrm{~mm}$ per $\mathrm{mm}^{2}$ region of interest in control). Basic metabolic phenotyping of adult mice shows no difference in body weight or fasting blood glucose levels $(5.0$ $\pm 0.2 \mathrm{mmol} / \mathrm{L}$ and $4.7 \pm 0.2 \mathrm{mmol} / \mathrm{L}$ in PIR-/- and control mice, respectively), but interestingly, preliminary assessment of pericyte function in vitro suggests enhanced proliferation of PIR-/pericytes.

Conclusions Pericyte-specific insulin receptor knockout affects developmental retinal angiogenesis in vivo. Sprouting and remodelling of the retinal vasculature are altered, associated with a reduction in pericyte coverage in adulthood, recapitulating some characteristics of proliferative diabetic retinopathy. The underlying molecular mechanism remains unclear, but these results indicate that adequate pericyte insulin signalling is important for pericyte function, and hence vascular stability.

\section{NOVEL THIENOPYRIDINES ARE POTENT ANTI-PLATELET DRUGS, INHIBITING PLATELET ACTIVATION, AGGREGATION AND SHOWING SYNERGY WITH ASPIRIN}

${ }^{1}$ Naif Binsaleh*, ${ }^{1}$ Catherine A. Wigley, ${ }^{1}$ Kathryn A. Whitehead, ${ }^{1}$ Daniel Moreno-Martinez, ${ }^{1}$ Sarah Daniels, 'Sarah Jones, ${ }^{2}$ Michelle van Rensburg, ${ }^{2}$ Lisa Pilkington, ${ }^{2}$ David Barker, ${ }^{1}$ Nina Dempsey-Hibbert. 'School of Healthcare Science, Manchester Metropolitan University, Manchester, UK, M1 5GD; ${ }^{2}$ School of Chemical Sciences, The University of Auckland, New Zealand

\subsection{6/heartjnl-2017-311726.185}

Background Management of Acute Coronary Syndromes (ACS) often involves the use of platelet inhibitors. The most commonly used drug, clopidogrel, belongs to a class of thienopyridine molecules which targets the $\mathrm{P}_{2} \mathrm{Y}_{12}$ receptor on platelets and it is commonly used in combination with the COX-1 inhibitor acetylsalicylic acid (ASA). However, the effect of these treatments is variable amongst patients, highlighting a need for a refinement of this class of $\mathrm{P}^{2} \mathrm{Y}_{12}$ inhibitor. The aim of this study was to assess the efficacy of six novel thienopyridine derivatives synthesised by our group by examining their potential as in-vitro inhibitors of platelet function.

Methods Healthy human platelets were isolated and incubated with novel thienopyridine compounds (DJ0081, DJ0199, DJ0021, DJ0206, DJ0171, DJ0097) $(10 \mu \mathrm{M}, 30 \mathrm{~min})$ prior to stimulation with ADP $(10 \mu \mathrm{M})$ and analysis of alpha granule secretion (CD62P expression), GPIIbIIIa activation (PAC1 expression) and platelet leukocyte aggregate (PLA) formation using flow cytometry. Furthermore, light transmission aggregometry (LTA) was used to assess ADP-stimulated aggregation after these treatments. Synergy with ASA $(30 \mu \mathrm{M})$ was also analysed by LTA following incubation with ASA and thienopyridine. All results were compared to ADP-stimulated samples and samples treated with clopidogrel $(10 \mu \mathrm{M}, 30 \mathrm{~min})$ prior to ADP stimulation.

Results All six novel compounds demonstrated a significant reduction in ADP-mediated platelet aggregation $(\mathrm{p}<0.001)$, CD62P expression $(\mathrm{p}<0.001)$, PAC1 expression $(\mathrm{p}<0.01)$ and PLA formation $(p<0.05)$. These compounds were also shown to enhance the inhibitory effects of ASA. DJ0171 and DJ0199 were particularly potent, displaying greater inhibitory effect than clopidogrel.

Conclusion The study demonstrates the potential for new thienopyridine compounds as modulators of platelet function and points to the possibility of future use in patients at risk of platelet hyperactivity and thrombosis.

\section{DEVELOPMENT OF A NOVEL SOFTWARE PACKAGE FOR HIGH-THROUGHPUT PROCESSING AND ANALYSIS OF CARDIAC OPTICAL MAPPING DATA}

Christopher O'Shea ${ }^{1,2,3},{ }^{2}$ Andrew Holmes, ${ }^{2}$ Ting Yue Yu, ${ }^{4}$ James Winter, ${ }^{5}$ Joao Correia, ${ }^{2}$ Paulus Kirchhof, ${ }^{2}$ Larissa Fabritz, ${ }^{3}$ Kashif Rajpoot, ${ }^{2}$ Davor Pavlovic. ${ }^{1}$ EPSRC Centre for Doctoral Training in Physical Sciences for Health, University of Birmingham, Birmingham,UK; ${ }^{2}$ Institute of Cardiovascular sciences, University of Birmingham, Birmingham, UK: ${ }^{3}$ School of Computer science, University of Birmingham, Birmingham, UK; ${ }^{4}$ Cardiovascular Division, The Rayne Institute, King's College London, UK; ${ }^{5}$ Cairn Research, Graveney Road, Faversham, UK

\subsection{6/heartjnl-2017-311726.186}

Background Optical mapping is a powerful research tool that is revolutionising study of cardiac electrophysiology. However, processing and analysis of optical mapping data is computationally challenging to design and implement, a difficulty further enhanced by novel camera technology providing higher temporal and spatial resolution. We have previously developed algorithms capable of objective processing and analysis of electrophysiological parameters acquired using a second-generation complementary metal-oxide semiconductor camera. ${ }^{1}$ Here we report development of improved algorithms packaged in a user-friendly graphical interface, capable of high-throughput processing and analysis of voltage and calcium optical mapping data from a wide spectrum of cameras. Functionality and processing speed is further improved through automated recognition of pacing frequency and analysis of activation and repolarisation of electrophysiological parameters at high spatiotemporal resolution $(200 \times 2400$ pixels; sampling rate $1 \mathrm{kHz}$ ). Processing options allow averaging of multiple beats as well as individual beat segmentation within the whole experimental trace, thus allowing for study of dynamic changes in key parameters such as action potential duration (APD), activation times, conduction velocity (CV) and phase mapping.

Methods and results We have compared how our software performs in terms of analysis and processing time with our 